\title{
CXCR4-Antagonist: Plerixafor verbessert die Mobilisierung in der autologen hämatopoetischen Stammzelltransplantation
}

\begin{abstract}
Bei der Mobilisierung von hämatopoetischen Stammzellen für die autologe Stammzelltransplantation können Patienten mit Multiplem Myelom oder Lymphom von dem CXCR4-Antagonisten Plerixafor $\left(\right.$ Mozobil $\left.^{\circledR}\right)$ profitieren: In Kombination mit dem Wachstumsfaktor G-CSF können mehr Patienten in weniger Apheresesitzungen die erforderliche Anzahl an CD34+ Stammzellen mobilisieren [1-2]. Dies macht die Apherese effizienter sowie besser planbar und ermöglicht letztendlich mehr autologe Transplantationen, so der Tenor der Experten bei einem Symposium, das im Rahmen des 31. Kongresses der International Society of Blood Transfusion (ISBT) sowie des 43. Kongresses der Deutschen Gesellschaft für Transfusionsmedizin und Immunhämatologie (DGTI) im Juni 2010 in Berlin stattfand [3].
\end{abstract}

Ziel der autologen Transplantation von Stammzellen ist es, die Dauer der Chemotherapie-induzierten Aplasie zu verkürzen sowie die behandlungsbedingte Morbidität und Mortalität zu reduzieren. Hauptindikationen sind Leukämien, lymphoproliferative Störungen, solide Tumoren und nichtmaligne Erkrankungen. So waren unter den insgesamt 16000 im Jahr 2008 in Europa durchgeführten autologen Stammzelltransplantationen ca. 13400 Patienten mit lymphoproliferativen Erkrankungen, 1400 Patienten waren an soliden Tumoren erkrankt und knapp 1000 litten an einer Leukämie [4]. «Die autologe Stammzelltransplantation bleibt dabei trotz der Einführung neuer Wirkstoffe wie Rituximab, Bortezomib, Thalidomid oder Lenalidomid eine wichtige Therapieoption v. a. bei Patienten mit hohem Rückfallrisiko oder, wenn mit herkömmlicher Therapie keine vollständige Remission erreicht werden kann», betonte Professor Dr. Christian Chabannon, Université de la Méditerranée, Aix-Marseille, Frankreich.

\section{G-CSF - ein wichtiger Meilenstein}

Bei der Entwicklung der Stammzelltransplantation stellte der Einsatz des rekombinanten Wachstumsfaktors G-CSF (granulocyte-colony stimulating factor) zur verstärkten Mobilisierung von Stammzellen aus dem Knochenmark in das periphere Blut einen wichtigen Meilenstein dar. Dies ermöglichte ab Mitte der 1980er Jahre den Ersatz von Knochenmarkstransplantaten durch Bluttransplantate. Da der Gehalt an Stammzellen im Transplantat den Erfolg der hämatopoe- tischen Stammzelltransplantation (HSZT) bestimmt, kann die Übertragung jedoch nur dann durchgeführt werden, wenn eine ausreichende Menge an CD34+ Zellen aus dem peripheren Blut gewonnen werden kann. Dabei gelten zwei Millionen CD34+ Zellen pro kg Körpergewicht als Minimum für eine erfolgreiche Transplantation, wünschenswert ist jedoch eine Zielzellzahl von fünf Millionen Stammzellen pro kg Körpergewicht. Deshalb galt in der Folge die besondere Aufmerksamkeit möglichen Prädiktoren für eine erfolgreiche Mobilisierung und Strategien, mit denen einer schlechten Mobilisierung begegnet werden kann. Das bisher zur Mobilisierung von Stammzellen in das periphere Blut eingesetzte G-CSF hat den Nachteil, dass der ideale Zeitpunkt für die Apherese sowie die Anzahl der erforderlichen Sitzungen schlecht vorhersagbar sind. Bei einigen Patienten können trotz mehrerer Sitzungen im Verlauf von mehreren Tagen unter G-CSF allein nicht genügend CD34+ Zellen für eine Transplantation gesammelt werden. «Für diese Patienten stehen seit kurzem echte Verbesserungen zur Verfügung», so Chabannon: Eine neue Generation leistungsfähiger Zellseparatoren wurde eingeführt und der CXCR4Antagonist Plerixafor zugelassen.

\section{Plerixafor-Wirkmechanismus: First In-Class-CXCR4-Inhibitor}

Blutstammzellen (CD34+ Zellen) werden im Knochenmark über die Interaktion zwischen dem Chemokin-Rezeptor CXCR4 und dem Chemokin SDF-1 $\alpha$ gebunden. Um eine ausreichende Menge an Blutstammzellen für die Transplantation aus dem peripheren Blut gewinnen zu können, muss die Verbindung zwischen Stammzelle und Nische gelöst werden. Anders als G-CSF, das indirekt wirkt, beeinflusst das «small molecule» Plerixafor spezifisch und direkt die Verbindung zwischen CXCR4 und SDF-1 $\alpha$. Durch die Blockade der Bindung werden mehr Blutstammzellen vom Knochenmark gelöst und in das periphere Blutsystem entlassen. Im Zusammenspiel mit G-CSF nehmen die Geschwindigkeit und Effektivität der Mobilisierung signifikant zu. Dies bestätigen u. a. Daten von Liles et al. [5], die Dr. Catarina Geraldes, Universitätskrankenhaus Coimbra, Portugal, präsentierte: Der Einsatz von Plerixafor zusätzlich zu einem fünftägigen $\mathrm{G}$ CSF-Regime führte im Zeitfenster zwischen

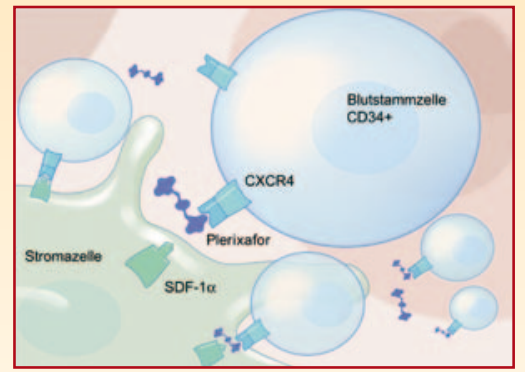

Abb. 1. Wirkmechanismus von Plerixafor

4 und 18 Stunden nach der Applikation zu einer signifikanten Erhöhung der peripheren CD34+Zellzahl $(n=10 ; p<0,05)$. Der Peak der CD34+ Zellen lag zwischen 10 und 14 Stunden nach der Plerixafor-Gabe.

\section{Zulassungsstudien: Signifikant bessere Mobilisierung bei Non-Hodgkin- Lymphom und Multiplem Myelom}

Die Fragestellung der von Geraldes präsentierten multizentrischen, randomisierten, doppelblinden, plazebokontrollierten Phase-IIIStudien [1-2] war es, Sicherheit und Wirksamkeit von Plerixafor plus G-CSF vs. GCSF-Monotherapie bei der Mobilisierung hämatopoetischer Stammzellen zur autologen Transplantation bei Patienten mit Non-Hodgkin-Lymphom (NHL, $n=298$ ) bzw. Multiplem Myelom (MM, $n=302$ ) zu untersuchen.

Mobilisierung bei Patienten mit Non-Hodgkin-Lymphom

In der NHL-Studie [1] wurden die Patienten nach der Randomisierung an acht aufeinanderfolgenden Tagen täglich mit $10 \mu \mathrm{g}$ GCSF pro kg Körpergewicht behandelt. Beginnend am Abend des vierten Tages erhielten die Patienten zusätzlich entweder 240 $\mu \mathrm{g}$ Plerixafor pro $\mathrm{kg}(\mathrm{n}=150)$ oder Plazebo ( $\mathrm{n}=148)$ für insgesamt vier Tage. Die Apheresesitzungen wurden am fünften Tag begonnen und bis einschließlich des achten Tages fortgeführt. Primärer Endpunkt der Untersuchung war der prozentuale Anteil der Patienten, bei denen $\geq 5 \times 10^{6}$ CD34+ Zellen pro $\mathrm{kg}$ in vier oder weniger Apheresesitzungen gesammelt werden konnten. Sekundäre Endpunkte waren der prozentuale Anteil der Patienten, die $\geq 2 \times 10^{6}$ 
CD34+ Zellen pro $\mathrm{kg}$ in vier oder weniger Apheresesitzungen erreichten, die Anzahl der Tage bis zum Anwachsen der Neutrophilen und Thrombozyten sowie die Transplantatbeständigkeit 100 Tage nach Transplantation.

90\% der Plerixafor-Patienten konnten transplantiert werden

65,6\% der Patienten unter Plerixafor erreichten in vier oder weniger Apheresetagen $\geq 5 \times 10^{6} \mathrm{CD} 34+$ Zellen pro $\mathrm{kg}$, gegenüber $24,2 \%$ in der Plazebo-Gruppe ( $<<0,0001$ ) (Abb. 2). Darüber hinaus erreichten in der Plerixafor-Gruppe bereits nach einem Apheresetag mehr Patienten (27,9\%) diese Zellzahl als in der Plazebo-Gruppe nach vier Tagen $(24,2 \%)$. Während $90 \%$ der Plerixafor-Patienten transplantiert werden konnten, lag dieser Endpunkt mit 55\% der Patienten in der Plazebo-Gruppe deutlich niedriger $(p<0,001)$. Die Gabe von Plerixafor plus G-CSF war sicher und gut verträglich.

\section{Mobilisierung bei Patienten mit Multiplem Myelom}

In der Zulassungsstudie für Plerixafor beim MM [2] erhielten die Patienten nach der Randomisierung an vier aufeinander folgenden Tagen täglich $10 \mu \mathrm{g}$ G-CSF pro $\mathrm{kg}$, ab dem fünften Tag zusätzlich täglich $240 \mu$ g Plerixafor pro $\mathrm{kg}(\mathrm{n}=148)$ oder Plazebo $(n=150)$. Auch in dieser Studie wurden die Apheresesitzungen am fünften Tag begonnen und, soweit notwendig, bis einschließlich des achten Tages fortgeführt. Primärer Endpunkt war der prozentuale Anteil der Patienten, die $\geq 6 \times 10^{6}$ CD34+ Zellen pro $\mathrm{kg}$ in zwei oder weniger Apheresesitzungen erreichten. Sekundäre Endpunkte waren unter anderem der prozentuale Anteil der Patienten, die $\geq 2 \times 10^{6}$ oder $\geq 6 \times 10^{6} \mathrm{CD} 34+$ Zellen pro $\mathrm{kg}$ in vier oder weniger Apheresesitzungen erreichten, die Anzahl der Tage bis zum Anwachsen der Neutrophilen und Blutplättchen

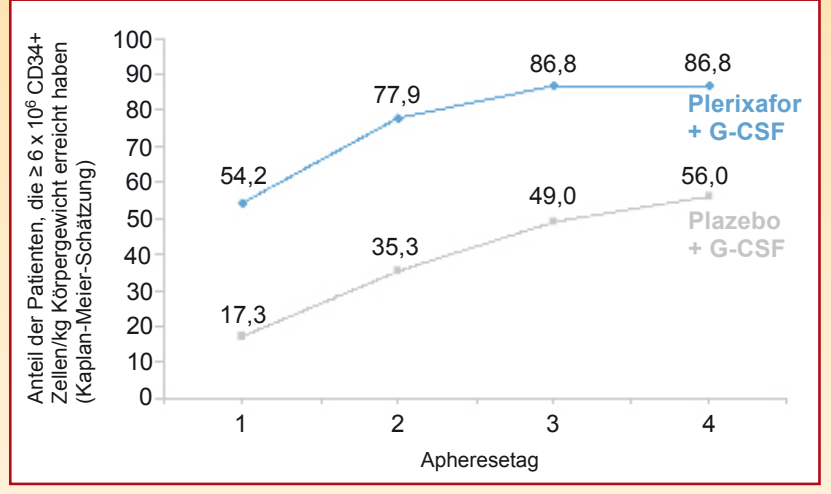

Abb. 3. Mobilisierung be Patienten mit Multiplen Myelom. sowie die Transplantatbeständigkeit 100 Tage nach Transplantation.

\section{Rasches Erreichen der Zielzellzahlen}

Mehr als doppelt so viele Patienten $(77,9 \%)$ aus der Plerixafor-Gruppe erreichten die geforderten $\geq 6 \times 10^{6} \mathrm{CD} 34+$ Zellen pro kg in zwei oder weniger Apheresetagen, gegenüber 35,3\% in der G-CSF-Monogruppe ( $\mathrm{p}<0,0001)$ (Abb. 3). Die Gabe von Plerixafor plus G-CSF war auch in dieser Untersuchung sicher und gut verträglich. Fazit der Zulassungsstudien ist Geraldes zufolge, dass die Kombination aus Plerixafor und GCSF der alleinigen Gabe von G-CSF zur autologen Stammzelltransplantation bei Patienten mit NHL und MM signifikant überlegen ist. Mit Plerixafor wurde die notwendige und optimale Menge an CD34+ Zellen für die Transplantation in weniger Apheresetagen erreicht. In beiden Studien hatte die Mobilisierung mit Plerixafor plus G-CSF keinen Einfluss auf das Engraftment (Neutrophile und Thrombozyten) sowie auf die Beständigkeit der Transplantate.

\section{Poor Mobilizer - Patienten, die zu wenig Stammzellen mobilisieren}

Damit eine autologe Stammzelltransplantation erfolgreich durchgeführt werden kann, ist ein Minimum an Stammzellen im Transplantat

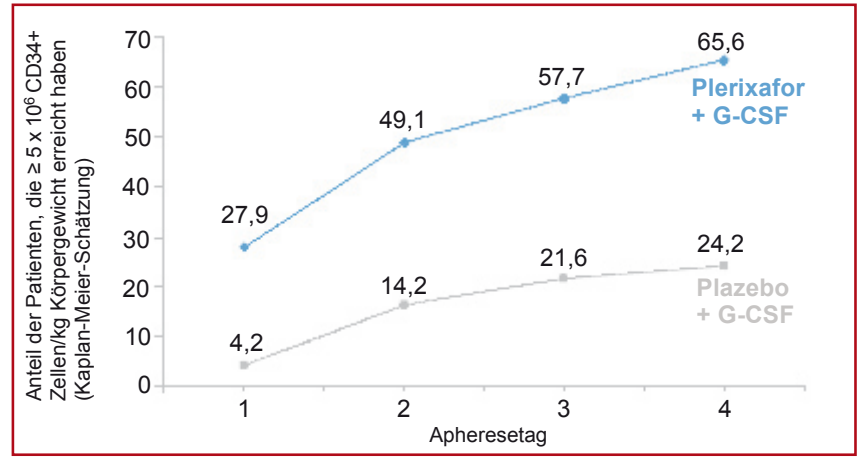

Abb. 2. Mobilisierung bei Patienten mit Non-HodgkinLymphom. erforderlich. PD Dr. Andreas Humpe, Universitäts-Krankenhaus Schleswig-Holstein, Kiel, präsentierte [3] dazu Daten von Giralt et al. [6], die für die Transplantation von Patienten mit MM eine minimale CD34+ Dosis von $4 \times$ $10^{6}$ Zellen pro $\mathrm{kg}$ und eine optimale Zellzahl von 8 bis $10 \times 10^{6}$ Zellen pro kg untersucht haben. Nach derzeitiger Auffassung liegt beim NHL die optimale CD34+ Zellzahl Humpe zufolge bei 4 bis $5 \times 10^{6}$ Zellen pro $\mathrm{kg}$.

\section{Versagerquoten variieren stark}

Diese Zahlen werden jedoch häufig nicht erreicht - betroffene Patienten werden als Poor Mobilizer bezeichnet. Professor Dr. Nina Worel, Universität Wien, Österreich, wies dabei auf die hohe Variabilität der nicht ausreichenden Mobilisierungen in der Literatur hin: Sie liegen je nach Studie beim MM zwischen 11 und 53\% und beim NHL bei etwa $5 \%$. In einer aktuellen Untersuchung gingen Pusic et al. [7] mit Hilfe einer retrospektiven Analyse der Frage nach, wie häufig die Mobilisierung nach einer Chemomobilisierung (Chemotherapie gefolgt von G-CSF) oder nach einem Steady-State-Regime mit G-CSF allein versagt: Von den 1040 Patienten, die eine autologe HSZT erhalten hatten, waren 5,9 bzw. 6,3\% der MM-Patienten und 22,9 bzw. 26,8\% der NHL-Patienten betroffen. Die Kombination mit einer Chemotherapie beeinflusste die Versagerquoten nicht.

Uneinheitliche Definitionen von fehlgeschlagener Mobilisierung

Hintergrund der stark variierenden Raten an Poor Mobilizern sind Worel zufolge neben einer Vielzahl verschiedener Mobilisierungsregime auch von Zentrum zu Zentrum unterschiedliche Definitionen eines fehlgeschlagenen Mobilisierungsversuchs. So werden als Kriterien für ein 
Therapieversagen u. a. folgende Parameter gewählt:

Zahl der zirkulierenden CD34+ Zellen

Nichterreichen der minimalen Zahl an CD34+ Zellen

Nichterreichen der optimalen CD34+ Zellzahl

keine Hochdosis-Chemotherapie möglich. Um für mehr Klarheit zu sorgen, hat die Arbeitsgruppe von Wuchter et al. [8] anhand einer retrospektiven Analyse bei 840 Patienten (602 MM, 238 NHL), verschiedene Gruppen von Poor Mobilizern ( $<20$ CD34+ Zellen/ $\mu$ l) definiert: 11-19 Zellen/ $\mu \mathrm{l}$ : «Borderline Poor Mobilizer», 6-10 Zellen/ $\mu$ : «Relative Poor Mobilizer» und $\leq 5$ Zellen/ $\mu$ : «Absolute Poor Mobilizer». Eine praxisnahe Definition der Poor Mobilizer hat auch die italienische Arbeitsgruppe Trapianto Midollo Osseo (GITMO) vorgeschlagen (siehe Kasten) [4].

\section{Suboptimale Mobilisierung - Risikofaktoren und Konsequenzen}

Besonders häufig ist Worel zufolge eine schlechte Mobilisierung von Stammzellen bei Patienten, die älter als 60 Jahre sind, oder unter bestimmten Grunderkrankungen leiden sowie bei vorangegangener Radio- und Chemotherapie, einer Behandlung mit Melphalan, Carmustin oder Fludarabin in der Anamnese oder unter neuen Induktionsstrategien (z.B. Lenalidomid beim MM). Kann keine ausreichende Zahl an CD34+ Zellen im Knochenmark mobilisiert werden, so erhöht sich die Zahl der Apheresetage; die Gewinnung von Knochenmark ist möglicherweise erforderlich oder die Transplantation kann unter Umständen überhaupt nicht durchgeführt werden.
Wiederholte Mobilisierungsversuche belasten darüber hinaus den Patienten, sind mit höherer Morbidität sowie Versagerquoten assoziiert und steigern den Ressourcenverbrauch. Der Gebrauch von Aphereseprodukten mit suboptimalem Ertrag kann dazu führen, dass die Stammzellen verspätet, nur teilweise oder gar nicht anwachsen. Das Risiko für Infektionen und/oder Blutungen ist ebenso erhöht wie das Risiko für Transfusionen.

Patienten profitieren vom Plerixafor-RescueProtokoll der Zulassungsstudien

Die von Worel präsentierten Rescue-Protokolle der beiden Zulassungsstudien von DiPersio [1-2] machen deutlich, dass Patienten, die unter GCFS allein nur unzureichend Stammzellen mobilisieren, von einer Plerixafor-Gabe profitieren: 10 Patienten der G-CSF/Plerixafor-Gruppe und 52 Patienten der Plazebo-Gruppe von den insgesamt 298 NHL-Patienten konnten in der Phase-III-Studie nicht ausreichend mobilisert und deshalb nicht transplantiert werden [9]. Im Rahmen des Rescue-Protokolls wurden sie mit G-CSF/Plerixafor behandelt. Anschließend war bei $60 \%$ der Patienten der ursprünglichen Verum-Gruppe und $88 \%$ der ursprünglichen Plazebo-Gruppe doch noch eine ausreichende Zellzahl zu erzielen und die Transplantation war möglich. Alle Plerixafor-Patienten mit MM (302) konnten transplantiert werden, bei sieben Patienten der Plazebo-Gruppe war die Mobilisierung nicht ausreichend. Nach Behandlung mit Plerixafor im Rahmen des Rescue-Protokolls konnten alle verbliebenen MMPatienten transplantiert werden. Worel zufolge ermöglicht die Verfügbarkeit von Plerixafor heute eine frühe Intervention bei nachgewiesenen und wahrscheinlichen Poor Mobilizern.

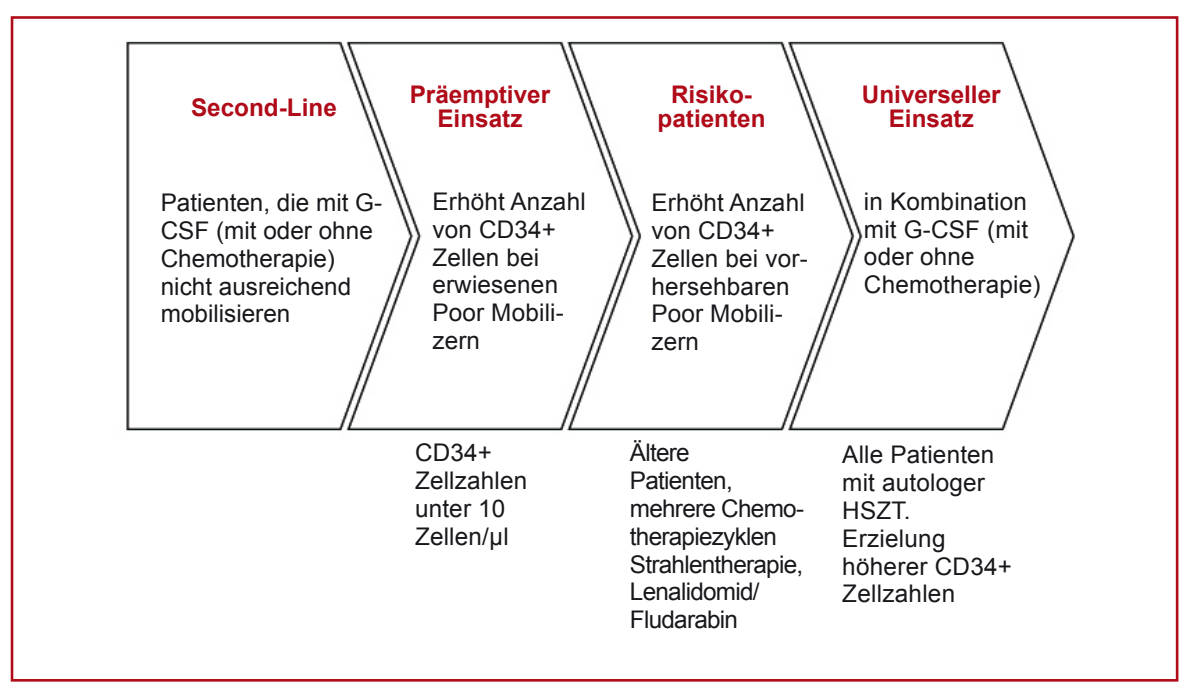

Abb. 4. Notwendigkeit zum Einsatz neuer Strategien für die autologe Stammzellmobilisierung.

\section{Poor Mobilizer - die GITMO-Definition}

Die Studiengruppe GITMO (Gruppo Italiano Trapianto Midollo Osseo) hat folgende Definitionen für Poor Mobilizer entwickelt [4]

\section{Belegte Poor Mobilizer:}

Patient mit MM oder NHL, der eine HSZT benötigt und der

- nach einer adäquaten Mobilisierung mit G-CSF Peak-Zellzahlen (CD34+) von < 20 Zellen/ $\mu$ am Tag 4 bis 6 nach dem Start der Mobilisierung mit G-CSF alleine oder nach Tag 18 bis 20 nach Chemotherapie und G-CSF zeigt oder

$-<2,5 \times 10^{6}$ gesammelte CD34+ Zellen $/ \mathrm{kg}$ Körpergewicht pro geplanter Stammzelltransplantation bei drei oder weniger Apheresesitzungen erreicht.

\section{Wahrscheinliche Poor Mobilizer:}

Patient mit MM oder NHL, der eine HSZT benötigt und der mindestens ein Hauptkriterium oder zwei Nebenkriterien erfüllt.

\section{Hauptkriterien:}

- vorangegangener Mobilisierungsversuch fehlgeschlagen

- extensive Radiotherapie auf Knochenmarksgewebe in der Anamnese

- Vortherapie kompletter Zyklen mit Melphalan, Fludarabin oder anderen Therapien, die die Stammzellmobilisierung beeinflussen können

Nebenkriterien:

- fortgeschrittene Erkrankung, z. B. nach zwei oder mehr zytotoxischen Therapielinien

- refraktäre Erkrankung

- extensive Knochenmarksbeteiligung bei der Mobilisierung

- Knochenmarkszellularität zum Zeitpunkt der Mobilisierung unter 30\%

- Alter über 65 Jahre

Sie plädierte deshalb für einen präemptiven Therapieansatz bei der Behandlung mit Plerixafor, um optimale Transplantationsraten zu erzielen. Für die Zukunft sieht Worel neben dem SecondLine-Einsatz von Plerixafor bei Patienten, die bereits einen fehlgeschlagenen Mobilisierungsversuch hinter sich haben, hier die wesentliche Einsatzmöglichkeit für den CXCR4-Antagonisten (Abb. 4).

\section{Maßgeschneiderte Strategien für die Sammlung von hämatopoetischen Stammzellen}

Ziel der Mobilisierung ist es, grundsätzlich einen ausreichenden bzw. optimalen Ertrag an peripheren Stammzellen mit einem Minimum an Apheresesitzungen $\mathrm{zu}$ erreichen. Humpe erklärte [3], dass dabei sowohl die Mobilisierungs- als auch die Sammelstrategien auf die individuellen Bedürfnisse des Patienten zugeschnitten sein sollten, und machte dies anhand eines individuellen Dosierungsschemas für Plerixafor deutlich 
(Abb. 5). Humpe unterstrich dabei, dass die CD34+ Zellausbeute bei der Apherese nicht nur von der Anzahl an CD34+ Zellen im peripheren Blut, sondern auch maßgeblich von der Dauer, vom Volumen und von der Fließgeschwindigkeit der Apherese ( $\mathrm{ml} / \mathrm{min})$ abhängt. Für einen optimalen Ertrag müssen diese Parameter jeweils angepasst werden. Als Benchmark gelten Humpe zufolge eine Apheresedauer zwischen 120 und $300 \mathrm{~min}$, die Fließgeschwindigkeit sollte zwischen 50 und $65 \mathrm{ml} / \mathrm{min}$ liegen. Abschließend fasste Humpe [3] zusammen: «Unter dem Einsatz von Plerixafor ist die Stammzellmobilisierung und -sammlung bei Patienten mit suboptimaler Mobilisierung besser planbar und kalkulierbar und ermöglicht oft erst die autologe Stammzelltransplantation.»

\section{Erfahrungen aus dem deutschen Compassionate-Use-Programm}

Insgesamt 60 Patienten, bei denen die Mobilisierung fehlgeschlagen war, wurden im Rahmen des deutschen CompassionateUse-Programms an 23 Zentren mit Plerixafor behandelt. Sie litten überwiegend an MM sowie NHL und erhielten $240 \mu \mathrm{g}$ Plerixafor pro kg s.c. 9-11 Stunden vor der geplanten Apheresesitzung. 78,3\% (47/60) erhielten an mindestens vier Tagen vor der Plerixafor-Gabe zusätzlich G-CSF. Bei $76,6 \%$ dieser Patienten (36/47) konnten $\geq$ $2,0 \times 10^{6} \mathrm{CD} 34+$ Zellen pro kg gesammelt werden, der mediane Zellertrag lag bei $3,35 \times 10^{6}$ CD34+ Zellen pro kg. Neun Pa-

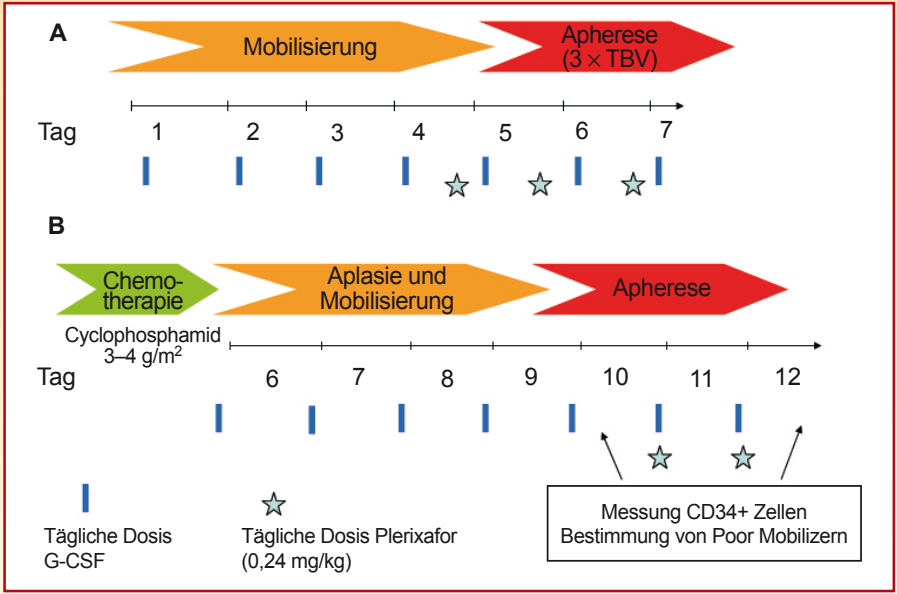

Abb. 5. Mobilisierungsschema nach DiPersio et al. (A) und beispielhaftes Mobilisierungsschema von Humpe (B) tienten erhielten entweder Plerixafor allein oder in Kombination mit G-CSF über weniger als vier Tage. Sie erzielten in der Apherese durchschnittlich $3,3 \times 10^{6}$ CD34+ Zellen pro kg. Es gab also keinen signifikanten Unterschied beim Zellertrag zwischen der viertägigen und der kürzeren Anwendungsdauer ( $\mathrm{p}=0,157)$. 47 Patienten erhielten Plerixafor plus G-CSF in Kombination mit einer Chemotherapie und erreichten durchschnittlich $3,28 \times 10^{6}$ CD34+ Zellen pro kg. 40 Patienten (66,7\%) konnten transplantiert werden und erreichten ein zeitgerechtes und stabiles Engraftment. Plerixafor war gut verträglich. Fazit der Autoren: Die Mobilisierung mit Plerixafor ist bei Poor Mobilizern sicher und führt bei $75 \%$ der Patienten zu einer ausreichenden CD34+ Ausbeute. Plerixafor

\begin{tabular}{|c|c|c|c|}
\hline & G-CSF-Monotherapie & G-CSF + Chemotherapie & G-CSF + Plerixafor \\
\hline Vorteile & $\begin{array}{l}\text { - geringerer Ressour- } \\
\text { cenverbrauch } \\
\text { - geringe Toxizität } \\
\text { - ambulante Patienten }\end{array}$ & $\begin{array}{l}\text { • höhere hämatopo- } \\
\text { etische Stammzellerträge } \\
\text { • weniger Apherese- } \\
\text { sitzungen } \\
\text { • Anti-Tumor-Aktivität } \\
\text { • vergleichbare Engraft- } \\
\text { ment-Charakteristiken }\end{array}$ & $\begin{array}{l}\text { - höhere hämatopoetische } \\
\text { Stammzellerträge } \\
\text { - gute Verträglichkeit } \\
\text { - weniger Apheresesitzungen } \\
\text { - gute Planbarkeit der } \\
\text { Apherese } \\
\text { - promptes und dauerhaftes } \\
\text { Anwachsen des } \\
\text { Transplantats } \\
\text { - einfache Handhabung } \\
\text { - Vorteile in Bezug auf } \\
\text { Logistik und Ressourcen }\end{array}$ \\
\hline Nachteile & $\begin{array}{l}\text { • kein Anti-Tumor- } \\
\text { Effekt } \\
\text { • höhere Versagerraten } \\
\text { bei der Mobilisierung } \\
\text { • signifikant erhöhte } \\
\text { Morbidität (z. B. } \\
\text { Knochenschmerzen, } \\
\text { Kopfschmerzen) }\end{array}$ & $\begin{array}{l}\text { - höhere Toxizität und } \\
\text { Hospitalisierungsraten } \\
\text { - Schädigung des } \\
\text { Knochenmarks möglich } \\
\text { • höherer Ressourcen- } \\
\text { verbrauch } \\
\text { - schlechtere } \\
\text { Vorhersagbarkeit }\end{array}$ & - kein Anti-Tumor-Effekt \\
\hline
\end{tabular}

Tab. 1. Verschiedene Mobilisierungsregime im Vergleich (nach Worel und Geraldes) war dabei auch bei Patienten wirksam, die an anderen (malignen) Erkrankungen litten, die eine Hochdosis-Chemotherapie und eine autologe Transplantation erfordern [10].

Monika Walter, München

\section{Referenzen}

1 DiPersio et al.: J Clin Oncol 2009;27:4767-73.

2 DiPersio et al.: Blood 2009;113:5720-26.

3 Symposium von Genzyme: «Emerging role of Plerixafor in stem cell mobilization and apheresis», im Rahmen des 31. Internationalen Kongresses der ISBT in Kooperation mit dem 43. Kongress der DGTI, 28. Juni 2010, Berlin.

4 Symposium von Genzyme: Vortrag von Lemoli $\mathrm{R}$ «Risk factors for poor mobilisation and clinical options for intervention», 36th Annual Meeting of the European Group for Blood and Bone Marrow Transplantation (EBMT), Wien, Österreich, 21. März 2010.

5 Liles et al.: Transfusion 2005;45:295-300.

6 Giralt et al.: Leukemia 2009;23:1904-12.

7 Pusic et al.: Biol Blood Marrow Transplant 2008;14:1045-56.

8 Wuchter et al.: Biol Blood Marrow Transplant 2010;16:1-10.

9 Micallef et al.: Biol Blood Marrow Transplant 2009;15:1578-86.

10 Hübel et al.: Bone Marrow Transplant 2010;45(S316): $\mathrm{P} 1005$.

Impressum

\section{CXCR4-Antagonist:}

Plerixafor verbessert die Mobilisierung in der autologen hämatopoetischen Stammzelltransplantation

PharmaForum in ONKologie 33 | 10 | 10

C 2010 by S. Karger Verlag für Medizin und Naturwissenschaften $\mathrm{GmbH}$

Wilhelmstraße 20A

79098 Freiburg, Deutschland

Mit freundlicher Unterstützung der Genzyme GmbH.

Der Verlag und die Herausgeber der Zeitschrift übernehmen keine Verantwortung für diese Rubrik. 